\title{
LA INSTITUCIONALIZACIÓN DE NORMAS SEGÚN EL DENOMINADOR COMÚN MÁS BAJO: LA NORMA ANTIGOLPISTA EN LAS NACIONES UNIDAS
}

\author{
LOWEST COMMON DENOMINATOR \\ NORM INSTITUTIONALIZATION: \\ THE ANTI-COUP NORM AT THE UNITED NATIONS

\section{L'INSTITUTIONNALISATION DE RÈGLES SUR LA BASE DU PLUS PETIT DENOMINATEUR COMMUN: LA NORME DES NATIONS UNIES FACE AUX COUPS D'ÉTAT}

\author{
Oisín TANSEY \\ King's College London \\ oisin.tansey@kcl.ac.uk
}

Resumen: Tras el fin de la Guerra Fría, Estados y organizaciones internacionales adoptaron progresivamente una nueva "norma antigolpista", la cual, sin embargo, se ha desarrollado de manera irregular y nunca se ha acogido o impulsado ni universal ni sistemáticamente. Este artículo examina la evolución desigual de la norma antigolpista mediante el seguimiento de la suerte que ha corrido en el sistema de las Naciones Unidas. El estudio muestra también cómo los Estados miembros han tratado de mantener la discreción precisa para reaccionar a casos de golpes de Estado particulares y cómo, en respuesta, los funcionarios de las Naciones Unidas en la Secretaría intentaron lograr la institucionalización de la norma, cambiando los estatutos y procedimientos internos de la organización para lidiar con los países golpistas. En su intento, buscaron la institucionalización de la norma del "denominador común más bajo", que consiste en procurar el grado más alto de institucionalización que pueda alcanzarse, aun si tal empeño implica aspirar a un nivel de progreso inferior al que, idealmente, complacería a los promotores de la norma.

Palabras clave: golpes de Estado, cumplimiento de las normas de las

Naciones Unidas.

Traducción de JORGE VALENZUELA 
Aвstract: After the end of the Cold War, states and international organizations increasingly adopted a new "anti-coup norm." However, this norm has evolved unevenly, and has never been universally embraced or consistently enforced. This article examines the uneven evolution of the anti-coup norm by tracing its fate within the United Nations system. It shows how member states have sought to retain discretion in how they respond to individual coup cases, and how, in response, UN officials within the Secretariat sought to pursue norm institutionalization by changing the UN's internal rules and procedures for dealing with coup countries. In doing so, they pursued the "lowest common denominator" norm institutionalization, which involves seeking to achieve the highest level of institutionalization that is achievable, even if it means aiming for a lower level of progress than norm promoters would ideally like.

Keywords: coups d'état, United Nations norm enforcement.

RÉsumé: Dès la fin de la guerre froide, peu à peu les États et les organisations internationales ont adopté une nouvelle norme "contre les coups d'État", dont le développement ne paraît pourtant pas constant et qui n'a pas été promue ni adoptée d'une façon universelle ni systématique. L'article examine cette évolution inégale de la norme, en suivant son parcours aux Nations Unies. L'analyse met en évidence que les États membres s'attachent à leur marge de pouvoir discrétionnaire lorsqu'ils doivent réagir à un coup d'État en particulier; au sein du Secrétariat, cela conduit les fonctionnaires des Nations Unies à essayer d'institutionnaliser la norme, en modifiant les règles et les procédures internes guidant l'organisation lorsqu'elle doit s'occuper de pays où un coup d'État a eu lieu. Dans ce but, les fonctionnaires ont misé sur l'institutionnalisation d'une norme "du plus petit dénominateur commun", qui consiste à encourager le plus haut niveau d'institutionnalisation possible, même s'il est inférieur à celui qui plairait -en tant que signe de progrès- aux partisans du principe.

Mots clefs: coups d'État, respect des normes des Nations Unies.

Traducción de Bernardo MABIRE

Fecha de recepción: mayo de 2017

Fecha de aceptación: abril de 2018 


\section{A}

L terminar la Guerra Fría, ${ }^{*}$ Estados y organizaciones internacionales se hicieron de cierta variedad de nuevas herramientas para promover normas y prácticas democráticas. Como parte de este impulso, muchos Estados adoptaron poderosos instrumentos legales para institucionalizar una nueva "norma antigolpista", que deslegitimaría y castigaría a los líderes que hubieran tomado el poder mediante un golpe de Estado. Las organizaciones regionales en África y las Américas lideraron el camino hacia la consolidación de un nuevo régimen de sanciones relacionado con el comportamiento de los golpes de Estado y demostraron su disposición a tomar medidas estrictas en contra de los Estados donde se hubiera violado la norma. ${ }^{1}$ Sin embargo, aunque cada vez hay más pruebas de que estas medidas han tenido importantes efectos en la disuasión de los intentos de golpe y en la reducción de la cantidad de golpes consumada con éxito, ${ }^{2}$ se ha tenido que luchar para que la norma goce del beneplácito general. Si bien ha habido momentos aislados en que la comunidad internacional ha sido unánime al defenderla (especialmente por medio de las resoluciones del Consejo de Seguridad de la onu y la Asamblea General), la norma aún no

* Se publica el artículo que aquí se ofrece por el acuerdo establecido entre Global Governance y Foro Internacional, según el cual ésta incluirá entre los propios un artículo de aquélla cada año y viceversa.

${ }^{1}$ T. Legler y T. Kwasi Tieku, "What Difference Can a Path Make? Regional Democracy Promotion Regimes in the Americas and Africa", Democratization 17, núm. 3, 2010, pp. 465-491; J. Heine y B. Weiffen, Twenty-first Century Democracy Promotion in the Americas: Standing Up for the Polity, Londres, Routledge, 2014; I. K. Souaré, "The AU and the Challenge of Unconstitutional Changes of Government in Africa", Institute for Security Studies Papers, núm. 197, 2009, pp. 1-13; J. Leininger, "Against All Odds: Strong Democratic Norms in the African Union", en T. A. Börzel y V. van Hüllen (eds.), Governance Transfer by Regional Organizations, Nueva York, Springer, 2015, pp.51-67, http://link.springer.com/chapter/10.1057/978 1137385642_3

${ }^{2}$ J. Powell, T. Lasley y R. Schiel, "Combating Coups d'État in Africa, 1950-2014", Studies in Comparative International Development 56, núm. 6, 2016, pp. 1-21, doi: 10.1007/s12116-015-9210-6 
se ha acogido universalmente ni se ha hecho cumplir a cabalidad. Varias organizaciones regionales esencialmente la han soslayado, en tanto que las Naciones Unidas se han mantenido "sistemáticamente inconsecuentes" en su trato con los gobiernos creados por un golpe de Estado. La norma antigolpista no es todavía verdaderamente universal. ${ }^{3}$

Como he adelantado en el resumen de este trabajo, mi propósito es examinar la descompasada evolución de la norma antigolpista al tiempo que doy seguimiento a su suerte y adversidades que ha enfrentado en el sistema de las Naciones Unidas. De este modo, no solamente ilustro el importante papel que desempeñan los Estados miembros de la onU y sus organismos, sino también un conjunto de procesos políticos que tiene implicaciones más amplias en la manera en que entendemos los esfuerzos de diversas partes del mundo por hacer cumplir las normas internacionales, aun cuando las contemporáneas rara vez se observen universalmente, sobre todo si se relacionan con el comportamiento de los gobiernos de los Estados nación en sus propias jurisdicciones. Con frecuencia, los actores internacionales que adoptan públicamente determinadas normas no responden con firmeza a las violaciones de alguna de ellas en particular o lo hacen de manera muy poco sistemática. ${ }^{4} \mathrm{~A}$ su vez, tal comportamiento frustra a aquellos que están verdaderamente comprometidos con la norma y, a menudo, los incita a buscar un mayor compromiso internacional con ella mediante nuevas formas de institucionalización. Esto da lugar a un ciclo que consta de fases bien calculadas, en que los artífices luchan para que se adopte una norma, sólo para atestiguar que no se observará y, luego, arremeter nuevamente con la intención de consolidar aún más la norma.

${ }^{3}$ O. Tansey, “The Fading of the Anti-coup Norm”, Journal of Democracy 28, núm. 1, 2017, 144-156, doi: 10.1353/jod.2017.0012

${ }^{4}$ M. Binder, "Humanitarian Crises and the International Politics of Selectivity", Human Rights Review 10, núm. 3, 2009, pp. 327-148, doi:10. 1007/s12142-009-0121-7 
La historia de la norma antigolpista en la onU ilustra la manera en que las organizaciones internacionales pueden ser el sitio clave de negociaciones sobre el cumplimiento de las normas y su desarrollo, además de sugerir que los burócratas internacionales individuales, que actúan en organizaciones intergubernamentales más amplias, cumplen con importantes funciones. Cuando los Estados miembros están unidos, pueden trabajar de manera conjunta para forjar nuevas condiciones vinculantes que se apliquen a todos los Estados. Sin embargo, cuando se carece de tal consenso, los promotores o artífices de normas que trabajan en organizaciones internacionales (or) suelen tener que buscar los medios para institucionalizar normas que no requieran acuerdos. En consecuencia, deben aspirar a lo que he dado en llamar la institucionalización de la norma del "denominador común más bajo”, que consiste, como adelanté líneas arriba, en procurar el grado más alto de institucionalización que pueda alcanzarse, aun si tal empeño implica aspirar a un nivel de progreso inferior al que, idealmente, complacería a los artífices o promotores de la norma. Estas estrategias de orden inferior pueden suponer la reforma (o creación) de reglas y procedimientos operativos comunes válidos para las agencias y el personal dentro de los órganos burocráticos de una oI, en tanto que dejan a los Estados miembros con autonomía para reivindicar políticas divergentes en las esferas intergubernamentales.

La suerte que corrió la norma antigolpista en las Naciones Unidas muestra cómo los funcionarios de este sistema intentaron compensar la falta de consenso entre los Estados miembros al tratar de hacerse con este tipo de institucionalización de bajo nivel, basándose principalmente en las enmiendas a las normas y procedimientos de las Naciones Unidas. Ante la ausencia de consenso entre los Estados miembros sobre la norma antigolpista, los promotores o artífices de normas dentro de la Secretaría lograron avanzar, procurando un cambio en un nivel más bajo de institucionalización e introduciendo nuevas reglas y procedimientos que 
se aplicarían al personal de las Naciones Unidas y que lograrían más coherencia entre los organismos burocráticos de su sistema.

El artículo se organiza del siguiente modo. Primero, examino la naturaleza del cumplimiento selectivo de normas e ilustro las estrategias de institucionalización de aquellas que pueden fomentar una mayor coherencia en su observancia. Luego, reviso la historia de la norma antigolpista en la ONU desde el final de la Guerra Fría y muestro el tratamiento eminentemente selectivo de ésta dentro del sistema de la onU. Finalmente, analizo las estrategias de la institucionalización de normas implementadas por el personal de la Secretaría de las Naciones Unidas y enfatizo la importante función de quienes promueven las normas en la burocracia de las Naciones Unidas.

\section{CUMPLIMIENTO DE NORMAS E INSTITUCIONALIZACIÓN}

Las normas son expectativas colectivas sobre los modelos de comportamiento que se definen en términos de derechos y obligaciones. ${ }^{5}$ Según el influyente postulado de Martha Finnemore y Kathryn Sikkink sobre el "ciclo de vida" de la evolución de las normas, éstas pasan por una serie de etapas antes de que se les adopte por completo y de que guíen el comportamiento en forma sistemática. La primera fase del ciclo es el surgimiento de la norma, en que los artífices o promotores (que a menudo tienen cargos en organizaciones internacionales formales) impulsan nuevos modelos normativos y persuaden a otros de adoptarlos. La segunda implica la "cascada de normas", en la que se alcanza cierto momento de inflexión y los países comienzan a adoptar rápidamente

${ }^{5}$ F. V. Kratochwil, Rules, Norms, and Decisions: On the Conditions of Practical and Legal Reasoning in International Relations and Domestic Affairs, vol. 2, Cambridge, University Press, 1991, p. 59; S. Percy, Mercenaries: The History of a Norm in International Relations, Oxford, University Press, 2007, p. 14. 
la nueva norma, incluso sin presión alguna. La tercera es la incorporación, en la que los actores se ajustan a la norma sin ponderación alguna. ${ }^{6}$

La medida en que las normas realmente rigen el comportamiento varía en parte según la fase de desarrollo normativo, y es menos probable que las nuevas se observen sistemáticamente que aquellas en fases posteriores del ciclo de vida de la norma. Antes de la plena incorporación de las normas, las respuestas que se ofrecen tras alguna violación de las normas internacionales suelen caracterizarse por ser inconsecuentes o selectivas. Como se define en gran parte de la bibliografía acerca del cumplimiento de las normas, la coherencia exige que los casos análogos se traten de la misma manera y que las violaciones de las normas que sean semejantes en su especie estén sujetas a respuestas también semejantes. ${ }^{7}$ Sin embargo, muchas normas internacionales bien desarrolladas se aplican de manera parcial e incongruente. Después de terminada la Guerra Fría, las organizaciones internacionales acogieron e institucionalizaron, cada vez con mayor ahínco, una variedad de normas diseñada para promover y proteger la democracia en todo el mundo, incluidas aquellas relacionadas con la apropiación del poder mediante golpes de Estado. ${ }^{8}$ Sin embargo, cuando se trata de normas relacionadas con la democracia, los Estados y las or han sido proclives, según las circunstancias, a tratar casos semejantes de maneras diferentes. Los actores internacionales castigan a los perpetradores del fraude elec-

${ }^{6}$ M. Finnemore, y K. Sikkink, "International Norm Dynamics and Political Change”, International Organization 52, núm. 4, 1998, pp. 887-917.

7 Binder, "Humanitarian Crises and the International...", art. cit., p. 329; D. Donno, Defending Democratic Norms: International Actors and the Politics of Electoral, Oxford, University Press, 2013, p. 77.

${ }^{8}$ D. Hawkins, "Protecting Democracy in Europe and the Americas", International Organization 62, núm. 3, 2008, pp. 373-403; J. C. Pevehouse, Democracy from Above: Regional Organizations and Democratization, Cambridge, University Press, 2005; Donno, Defending Democratic Norms, op. cit. 
toral en algunos casos, pero no en otros. ${ }^{9}$ La represión violenta de las fuerzas de oposición a veces recibe sanciones internacionales (como en el caso de Zimbabwe), mientras que violaciones análogas en otros lugares merecen, comparativamente, menos atención internacional (tal es el caso de Azerbaiyán). ${ }^{10}$ Los golpes de Estado han sido condenados y castigados en ciertos ámbitos, pero subestimados, e incluso celebrados, en otros. ${ }^{11}$

Una manera, entre otras, de superar estas modalidades de incongruencia es por medio del proceso de institucionalización de las normas, que conlleva una mayor regulación del comportamiento de actores clave. ${ }^{12}$ Como lo señalan Finnemore y Sikkink, "la institucionalización contribuye en gran medida a la posibilidad de una cascada de normas, ya que aclara qué es exactamente la norma y qué constituye una violación (a menudo, una cuestión de desacuerdo entre los actores) y detalla procedimientos específicos mediante los cuales los líderes normativos coordinan la desaprobación y las sanciones por incumplir la norma". ${ }^{13}$ La institucionalización contribuye a la claridad respecto de los factores desencadenantes para la aplicación y los tipos de medidas que para ello deben utilizarse; por lo tanto, al menos en teoría, facilita

${ }^{9}$ Donno, op. cit., p. 77; véase también J. Kelley, "D-minus Elections: The Politics and Norms of International Election Observation", International Organization 63, núm. 4, 2009, pp. 765-787, doi: 10.1017/S00208183 09990117

${ }^{10}$ E. M. Hafner-Burton, S. D. Hyde y R. S. Jablonski, "When Do Governments Resort to Election Violence?" British Journal of Political Science 44, núm. 1, 2013, pp. 1-31.

11 O. Tansey, "The Limits of the 'Democratic Coup' Thesis: International Politics and Post-coup Authoritarianism”, Journal of Global Security Studies 1, núm. 3, 2016, pp. 220-234.

12 J. Goldstein, M. Kahler, R. O. Keohane, A.-M. Slaughter, "Introduction: Legalization and World Politics”, International Organization 54, núm. 3, 2000, pp. 387, doi: 10.1162/002081800551262.

13 Finnemore y Sikkink, "International Norm Dynamics", art. cit., p. 900 . 
respuestas más coherentes con los comportamientos que violan las normas.

Las normas pueden institucionalizarse en la política internacional mediante un conjunto de instrumentos que incluye desde acuerdos y compromisos informales hasta estatutos y procedimientos más formales, además de, en última instancia, disposiciones legales obligatorias. Si las normas se han institucionalizado con base en una eminente y férrea legalización, deberíamos esperar grados inferiores de incongruencia en varios aspectos a medida que la severidad de las reglas limita el margen de maniobra de los Estados. ${ }^{14}$ Por el contrario, si las normas no se han legalizado plenamente y no alcanzan la categoría de normas imperativas, deberíamos esperar mayores niveles de cumplimiento selectivo. La legalización, para ser sólida, exige de notable consenso internacional y de voluntad política entre un gran número de Estados, lo que es difícil de lograr si las normas en cuestión son políticamente delicadas o controvertidas. Cuando se trata de temas como la libertad política y la democracia, ante los cuales el consenso normativo disminuye y la impugnación de normas se vuelve una característica peculiar de los debates internacionales, puede esperarse que se susciten distintas formas de incoherencia. ${ }^{15}$

Sin embargo, incluso cuando la legalización es relativamente laxa y el consenso general, una posibilidad remota, todavía queda una variedad de estrategias de institucionalización de las que los promotores de normas pueden valerse para reducir la falta de congruencia en el cumplimiento de las normas. En organizaciones internacionales como las $\mathrm{Na}$ ciones Unidas, los miembros individuales de la burocracia pueden ayudar a compensar la falta de acuerdos entre los Estados miembros mediante la búsqueda de modificaciones

${ }^{14}$ K. W. Abbott, R. O. Keohane, A. Moravcsik, A.-M. Slaughter y D. Snidal, "The Concept of Legalization", International Organization 54, núm. 3, 2000, pp. 401-419, doi: 10.1162/002081800551271

15 T. Carothers, "The Backlash Against Democracy Promotion", Foreign Affairs 85, núm. 2, 2006, pp. 55-68; A. Cooley, "Countering Democratic Norms”, Journal of Democracy 26, núm. 3, 2015, pp. 49-63. 
en las reglas que afecten al personal de or en lugar de a los Estados miembros de éstas. Tales medidas destacan la capacidad de las or para operar libremente, al margen de sus Estados miembros. Una parte importante de los estudiosos de las or ha observado que la presencia de una burocracia supranacional es fuente esencial para su autonomía, toda vez que las agencias y los actores pueden ejercer autoridad e influencia, independientemente de los mandatos que les otorgan los Estados miembros. ${ }^{16}$

El análisis de la función de los burócratas de las oi permite identificar los métodos con que las normas a veces se institucionalizan, a puerta cerrada, en éstas. Aquellos que trabajan en las secretarías de or suelen ser actores políticos comprometidos con la promoción y el avance de normas decisivas. Por lo tanto, los burócratas pueden, por su propio derecho, actuar como emprendedores de la norma y tratar de minimizar la incongruencia con que su propia organización responde a las violaciones de la norma. Si bien los burócratas de oI son incapaces de lograr formas de institucionalización legalizada y obligatoria que requieren el consenso y el acuerdo de los Estados miembros, aun así, pueden introducir reglas y procedimientos en su organización de manera que ayuden a superar los tipos de contradicción que surgen de la falta de respuestas coordinadas en una organización internacional. Estas reglas y procedimientos pueden desarrollarse independientemente de los Estados miembros e implicar esfuerzos para dirigir las respuestas de diferentes agencias dentro de la organización más amplia, así como coordinar actividades con organizaciones externas. Los burócratas internacionales en

${ }^{16}$ K. W. Abbott y D. Snidal, "Why States Act Through Formal International Organizations", Journal of Conflict Resolution 42, núm. 1, 1998, pp. 3-32, doi: 10.1177/0022002798042001001; Y. Z. Haftel y A. Thompson, "The Independence of International Organizations: Concept and Applications", Journal of Conflict Resolution 50, núm. 2, 2006, pp. 253-275, doi: 10.1177/0022002705285288; M. Barnett y M. Finnemore, Rules for the World: International Organizations in Global Politics, Ithaca, Cornell University Press, 2004. 
la Secretaría de las Naciones Unidas han participado en la institucionalización de reglas y normas en una amplia variedad de áreas temáticas, incluidas la prevención de conflictos, ${ }^{17}$ la promoción de la democracia, ${ }^{18}$ la igualdad de género ${ }^{19}$ y la gestión de la política científica. ${ }^{20}$ Como se explica detalladamente líneas abajo, en el caso de los burócratas de la norma antigolpista dentro de la Secretaría, se buscó obtener respuestas más congruentes y predecibles al quebrantamiento de las normas, incluso sin el apoyo de los Estados miembros, modificando las reglas vigentes hasta entonces e ideando nuevos procedimientos.

Hasta cierto punto, estos esfuerzos equivalen a la institucionalización del denominador común más bajo, es decir, que los actores involucrados desearían alcanzar peldaños más elevados en la escala de la institucionalización, pero no están en condiciones de hacerlo a menos que haya consenso y apetencia política entre los Estados miembros. En su lugar, se valen de su autonomía, a menudo relacionada con la capacidad para crear nuevas reglas y procedimientos que guíen las agencias y al personal de las or, pero que de ninguna manera orientan ni constriñen a los Estados miembros. De este modo, pueden esforzarse por superar

17 A. Björkdahl, "Promoting Norms Through Peacekeeping: UnPREDEP and Conflict Prevention", International Peacekeeping 13, núm. 2, 2006, pp. 214-228, doi: 10.1080/13533310500437613

${ }^{18}$ S. Rushton, "The UN Secretary-General and Norm Entrepreneurship: Boutros Boutros-Ghali and Democracy Promotion", Global Governance: A Review of Multilateralism and International Organizations 14, núm. 1, 2008, pp. 95-110; K. Haack, The United Nations Democracy Agenda: A Conceptual History, Oxford, University Press, 2011.

${ }^{19}$ M. L. Krook y J. True, "Rethinking the Life Cycles of International Norms: The United Nations and the Global Promotion of Gender Equality", European Journal of International Relations 18, núm. 1, 2012, pp. 103-127.

${ }^{20}$ M. Finnemore, "International Organizations as Teachers of Norms: The United Nations Educational, Scientific, and Cultural Organization and Science Policy", International Organization 47, núm. 4, 1993, pp. 565597, doi: 10.1017/S0020818300028101 
algunas modalidades de incoherencia en la aplicación mientras se reconcilian con su incapacidad para eliminar todas las formas.

En este artículo, ilustro cómo el personal de la onU trató de institucionalizar aún más la norma contra el golpe de Estado en respuesta a las incongruencias evidentes que caracterizaron la aplicación de tal norma. Si bien tratar de conseguir la legalización de la norma no era opción, los funcionarios de la onU intentaron enmendar las normas y procedimientos organizativos vigentes para lograr mayor congruencia en las respuestas de los organismos y el personal de la organización.

Cumplimiento inconsecuente De la nORMA ANTIGOLPISTA DE LA ONU

Desde que concluyó la Guerra Fría, el asunto concerniente a los golpes de Estado ha constituido el propósito fundamental de los esfuerzos internacionales para promover formas democráticas de gobierno, y la prohibición internacional contra el derrocamiento irregular de los gobiernos en turno, particularmente el de aquellos elegidos democráticamente se ha convertido en elemento esencial de la agenda normativa mundial. Sin embargo, la norma contra el golpe de Estado ha experimentado una suerte diversa en la oNU, donde, en última instancia, a una aceptación inicial de la norma siguió un enfoque por demás incongruente.

El auge de la norma contra el golpe de Estado ha sido más evidente en dos de las principales organizaciones regionales, la Unión Africana (UA) y la Organización de los Estados Americanos (OEA). En las últimas décadas, ambas organizaciones han incorporado instrumentos legales cada vez más sólidos para castigar gobiernos erigidos a partir del golpe de Estado, y cada una ha adoptado políticas de cumplimiento de las normas bastante congruentes (incluidas las 
suspensiones de los Estados miembros). ${ }^{21}$ Por el contrario, las Naciones Unidas han adoptado un enfoque selectivo sobre la norma antigolpista, a veces cobijándola, pero soslayándola en la mayoría de los casos. La perspectiva de la organización acerca de los países que experimentaron golpes de Estado ha sido más ostensible en la acción del Consejo de Seguridad luego de consumada la Guerra Fría. ${ }^{22}$ A partir de finales de los años ochenta, el Consejo comenzó por desempeñar un papel mucho más riguroso y, a veces, intervencionista en las políticas internas de sus Estados miembros. ${ }^{23}$ Este nuevo activismo incluyó un compromiso gradual con la promoción de normas y prácticas democráticas. ${ }^{24}$ Sin embargo, después de que en un principio parecía acoger la norma antigolpista en los albores de la década de 1990, el Consejo de Seguridad finalmente adoptó un enfoque eminentemente selectivo, con lo que demostró la gran incongruencia que llevaba aparejado el hecho de responder sólo a un reducido número de golpes de Estado en

${ }^{21}$ Souaré, "The AU and the Challenge of Unconstitutional Changes..., art. cit.; L. Nathan, "Trends in Mediating in Africa Coups, 20002015", investigación presentada en la International Studies Association Annual Convention, 2016; Heine y Weiffen, Twenty-first Century Democracy Promotion..., op. cit.

${ }^{22}$ La Asamblea General de las Naciones Unidas rara vez ha actuado en respuesta a los golpes de Estado, aunque las excepciones incluyen Haití, en 1991, y Honduras, en 2009, cuando la Asamblea condenó el derrocamiento de los gobiernos electos. Véase Asamblea General de las Naciones Unidas, Res. 46/7, 11 de octubre de 1991; Asamblea General de las Naciones Unidas, Res. 63/301, 1 de julio de 1999.

${ }^{23}$ S. von Einsiedel, D. M. Malone y B. Stagno Ugarte, The UN Security Council in the 21st Century, Boulder: Lynne Rienner, 2015, p. 5.

${ }^{24}$ C. C. Joyner, "The United Nations and Democracy", Global Governance 5, núm. 3, 1999, pp. 333-357; N. D. White, "The United Nations and Democracy Assistance: Developing Practice Within a Constitutional Framework", en Peter Burnell (ed.), Democracy Assistance: International Co-operation for Democratization, Londres, Frank Cass, 2000, pp. 67-89; C. E. Lombardo, "The Making of an Agenda for Democratization: A Speechwriter's View", Chicago Journal of International Law 2, núm. 1, 2001, pp. 253-266. 
el periodo posterior a la Guerra Fría. Así pues, reveló la disyunción entre su retórica normativa y el comportamiento operativo característico de la conducta de las Naciones Unidas en varios ámbitos. ${ }^{25}$

Una de las primeras pruebas de un Consejo de Seguridad más expansionista e intervencionista se llevó a cabo tras el derrocamiento del gobierno elegido democráticamente de Bertrand Aristide, en Haití. Aunque la Asamblea General condenó rápidamente el golpe, al principio el Consejo de Seguridad no logró tomar ninguna medida, en parte porque China tenía recelos de ampliar las facultades del Consejo para incluir temas que tradicionalmente se consideraban vinculados a la política interna. ${ }^{26}$ Sin embargo, después de la sostenida inestabilidad política en Haití y del surgimiento de una crisis de refugiados que afectó a Estados Unidos, en 1993 el Consejo aludió a circunstancias "únicas y excepcionales" y autorizó sanciones económicas contra el régimen militar en virtud del capítulo vir del Acta constitutiva de la ONu. ${ }^{27}$ Después de que las autoridades haitianas continuaran desafiando la presión internacional, el Consejo tomó otra resolución del capítulo viI en 1994, con base en la cual se autorizaron "todos los medios necesarios para facilitar la partida de Haití de los dirigentes militares" y lograr "el pronto regreso del Presidente legítimamente electo y la restauración de las autoridades legítimas del Gobierno de Haití". ${ }^{28}$ Bajo la amenaza de una inminente intervención dirigida por Estados Unidos, los líderes militares cedieron y Aristide fue restituido en el poder.

${ }^{25}$ S. von Billerbeck, "Local Ownership and UN Peacebuilding: Discourse Versus Operationalization”, Global Governance: A Review of Multilateralism and International Organizations 21, núm. 2, 2015, pp. 299-315.

${ }^{26}$ S. Chesterman, Just War or Just Peace? Humanitarian Intervention and International Law, Oxford, University Press, 2003, p. 152.

${ }^{27}$ Einsiedel, Malone y Ugarte, The UN Security Council in the Twenty-first Century, op. cit., p. 5.

${ }^{28}$ Véase Consejo de Seguridad de la onu, Res. S/REs/940, 31 de julio de 1994. 
A tan enérgica reacción en contra del golpe de Estado en Haití siguió, en 1997, una respuesta no menos categórica de soldados rebeldes al derrocamiento del presidente electo de Sierra Leona. Al igual que en el caso de Haití, el Consejo de Seguridad exigió la restitución de las autoridades electas y determinó que los incidentes constituían una amenaza para la paz y la seguridad internacionales. ${ }^{29}$ La Resolución 1132 del Consejo de Seguridad también autorizó a la Comunidad Económica de los Estados de África Occidental (CEDEAO) para que interrumpiera los suministros de ayuda extranjera a los líderes golpistas, además de validar implícitamente el uso de la fuerza de la CEDEAo en contra del, hasta ese momento, nuevo régimen. Después de una escalada en la aplicación regional de las leyes, el expresidente Ahmad Tejan Kabbah regresó al poder en 1998.

Ambos casos pusieron de relieve una nueva disposición del Consejo de Seguridad para autorizar medidas coercitivas que penalizaran a los líderes golpistas, y en sus resoluciones puso un gran énfasis en la necesidad de restablecer los gobiernos legítimos y democráticamente electos. La democracia estaba desempeñando en las resoluciones del Consejo de Seguridad un papel que no había tenido anteriormente, y se estaban autorizando medidas de aplicación imperativas para revertir los golpes de Estado de modos inusitados incluso unos años antes. Sin embargo, estos episodios no supusieron la aceptación más amplia de la norma antigolpista dentro de la ONU, y las contundentes medidas que se tomaron en Haití y Sierra Leona demostraron ser excepciones, en lugar de la regla. Jonathan Powell y Clayton Thyne identificaron cuarenta y cuatro golpes de Estado exitosos entre 1991 y 2018, ${ }^{30}$ pero fue notable la falta de firmeza del Consejo de Seguridad en la aplicación de la norma antigolpista, al responder

${ }^{29}$ Véase Consejo de Seguridad de la onu, Res. S/RES/1132, 8 de octubre de 1997.

${ }^{30}$ J. Powell y C. Thyne, "Global Instances of Coups from 1950 to 2010: A New Dataset", Journal of Peace Research 48, núm. 2, 2011, pp. 249259, doi: $10.1177 / 0022343310397436$ 
sólo a una mínima cantidad de éstos. Aparte de los casos de Haití y Sierra Leona, el Consejo incluyó referencias explícitas a los golpes de Estado en sólo un reducido número de sus resoluciones, como el caso de Burundi, en 1996, y el de Guinea-Bisáu en 2012. ${ }^{31}$ Muchos otros casos se trataron únicamente en las declaraciones presidenciales del Consejo de Seguridad y, por lo tanto, no tienen la categoría de resoluciones del Consejo (por ejemplo, Camboya en 1997 y Mauritania en 2008)..$^{32}$

El Consejo también mostró variedad en la retórica con la que respondió a los golpes de Estado. En ciertos casos, calificó los acontecimientos en cuestión como "golpes", sin dejar lugar a la ambigüedad, mientras que en otros evitó el término. También hubo situaciones en que "condenó" algún golpe de Estado, mientras que en otras sólo expresó "preocupación”. Por lo tanto, las respuestas del Consejo fueron en ocasiones enérgicas y contundentes, complementando su retórica condenatoria del golpe de Estado hasta con énfasis tipográfico (reprobó los actos con términos destacados en "negrita"), como hizo en Guinea-Bisáu en 2012 y Mauritania en 2008.33 Alternativamente, cuando deseaba conducirse con más cautela, utilizaba términos más ambiguos o imprecisos, como la ocasión en que se declaró "profundamente preocupado por los acontecimientos recientes" a raíz del golpe de Estado de Camboya en 1997. ${ }^{34}$

El Consejo también se mostró inconsecuente en las demandas que presentó a los líderes golpistas recién instalados.

31 Consejo de Seguridad de la onu, Res. s/Res/1072, 30 de agosto de 1996; Consejo de Seguridad de la onu, Res. s/REs/2048, 18 de mayo de 2012.

32 Consejo de Seguridad de la onu, Declaración de la Presidencia S/PRst/1997/37, 11 de julio de 1997; Consejo de Seguridad de la onU, Declaración de la Presidencia s/Prst/2008/30, 19 de agosto de 2008.

33 Consejo de Seguridad de la onu, Declaración de la Presidencia S/PRst/2012/15, 21 de abril de 2012; Consejo de Seguridad de la onu, Declaración de la Presidencia s/Prst/2008/30, 19 de agosto de 2008.

34 Consejo de Seguridad de la onu, Declaración de la Presidencia S/PRST/1997/37, 11 de julio de 1997. 
Su opción más contundente fue exigir su salida y la restauración o reincorporación del gobierno anterior, que solicitó para Haití en 1994, ${ }^{35}$ para Sierra Leona en 1997,36 para Fiji en $2006{ }^{37}$ y para Guinea-Bisáu en 2012. ${ }^{38}$ En otros casos, el Consejo simplemente pidió la "restauración del orden constitucional”, sin solicitar expresamente la restitución de los líderes expulsados, como ocurrió con Burundi en 1996, Guinea-Bisáu en 2003 y Mauritania en 2008. ${ }^{39}$

En la mayoría de los casos posteriores a 1991, sin embargo, el Consejo simplemente paso por alto los golpes de Estado y no emitió resolución o declaración presidencial alguna. Estos casos comprenden el periodo posterior a la Guerra Fría e incluyen a Qatar en 1995, Pakistán en 1999, Fiji en 2000, Tailandia en 2006 y 2014, y Egipto en 2013. Si bien el Consejo ha mostrado incongruencias en el alcance de la aplicación de la ley que procuró en aquellos casos en que emitió una respuesta, el indicador más claro de su tímida admisión de la norma reside en el silencio que dio como respuesta a la mayoría de los golpes de Estado que vinieron luego de consumada la Guerra Fría. ${ }^{40}$

35 Consejo de Seguridad de la onu, Res. s/Res/940, 31 de julio de 1994.

${ }^{36}$ Consejo de Seguridad de la onu, Res. s/Res/1132, 8 de octubre de 1997.

37 Comunicado de prensa del Consejo de Seguridad de la onU, sc/8894, 7 de diciembre de 2006.

${ }^{38}$ Consejo de Seguridad de la onu, Declaración de la Presidencia S/PRST/2012/15, 21 de abril de 2012.

${ }^{39}$ Consejo de Seguridad de la ONU, Res. s/REs/1072, 30 de agosto de 1996; Comunicado de prensa del Consejo de Seguridad de la onU sobre Guinea-Bisáu sc/7873, 15 de septiembre de 2003; Consejo de Seguridad de la ONu, Declaración de la Presidencia s/PRST/2008/30, 19 de agosto de 2008.

${ }^{40}$ El Consejo de Seguridad de la onu ha emitido resoluciones o declaraciones presidenciales (sus dos formas de declaración más fuertes) que invocan explícitamente la norma antigolpista en sólo siete casos desde 1991: el golpe de Estado en Haití en 1991 (múltiples declaraciones, incluida la Resolución 940); Burundi, en 1996 (Resolución 1072); Sierra Leona, en 1997 (múltiples declaraciones, incluida la Resolución 1132); 
Las causas de esta incongruencia son diversas. Los enfoques para comprender por qué el Consejo actúa de la manera en que lo hace tienden a contrastar las motivaciones normativas y basadas en el interés de los miembros del Consejo. ${ }^{41} \mathrm{Si}$ el Consejo se apegara a consideraciones normativas, esperaríamos verlo reaccionar con mayor firmeza ante los golpes que derrocan a líderes democráticamente electos, ya que la norma antigolpista hace mayor hincapié en prohibir los golpes que amenacen los regímenes democráticos. Hay algunas evidencias que sugieren que el Consejo se toma más en serio este tipo de golpes, y los casos en que se ha expresado con mayor contundencia han supuesto la destitución de los líderes electos. ${ }^{42}$ Sin embargo, el Consejo también ha soslayado el derrocamiento de muchos líderes elegidos democráticamente, entre ellos Nawaz Sharif, en Pakistán, en 1999; Thaksin Shinawatra, en Tailandia, en 2006, y Mohamed Morsi, en Egipto, en 2013.

Si bien las consideraciones normativas desempeñan cierta función cuando el Consejo de Seguridad toma sus decisiones, son insuficientes para explicar el patrón general y, por lo tanto, se vuelve necesario considerar motivaciones más estratégicas e impulsadas por los intereses en su seno. En un estudio sobre los determinantes en las decisiones que toma el

Camboya, en 1997 (Declaración de la Presidencia s/PRST/1997/37); Mauritania, en 2008 (Declaración de la Presidencia S/PRST/2008/30); Mali, en 2012 (múltiples declaraciones, incluida la Declaración de la Presidencia S/PRST/2012/7) y Guinea-Bisáu (múltiples declaraciones, incluida la Resolución 2048).

${ }^{41}$ K. Beardsley y H. Schmidt, "Following the Flag or Following the Charter? Examining the Determinants of UN Involvement in International Crises, 1945-20021", International Studies Quarterly 56, núm. 1 (2012): 33-49; Martin Binder, "Paths to Intervention: What Explains the Un's Selective Response to Humanitarian Crises?" Journal of Peace Research 52, núm. 6, 2015, pp. 712-726, doi: 10.1177/0022343315585847

42 D. Shraga, "The Security Council and Human Rights -From Discretion to Promote to Obligation to Protect", en B. Fassbender (ed.), Securing Human Rights? Achievements and Challenges of the UN Security Council, Oxford, University Press, n.d., pp. 16-17. 
Consejo acerca de la intervención en casos de crisis humanitaria, Martin Binder descubrió que las consideraciones normativas deben combinarse teniendo en cuenta otros tres factores: la participación previa de la onU en un caso, el alcance de los efectos derivados de una crisis y la fuerza de las condiciones del objetivo potencial. ${ }^{43}$ Cada uno de estos factores ayuda a explicar el enfoque no sistemático de la onU para hacer cumplir la norma antigolpista. La participación previa de la onu contribuye a dar cuenta de la voluntad del Consejo de afrontar los golpes en varios casos. Por ejemplo, la ONU había participado en la observación de los comicios de 1990 en Haití que llevaron a la elección del presidente Aristide antes de su derrocamiento en el golpe de 1991. Del mismo modo, la contundente respuesta del Consejo de Seguridad a los golpes de Estado en Sierra Leona (1997) y Guinea-Bisáu (2012) debe considerarse a la luz de los esfuerzos previos de la onU por resolver conflictos civiles en estos casos. También era más probable que algunos golpes atrajeran la atención del Consejo si había altos riesgos de desbordamiento y efectos colaterales, además de una mayor inseguridad regional. La evolución de la respuesta del Consejo al golpe de Estado en Haití a principios de la década de 1990 estuvo indisolublemente vinculada a la crisis de refugiados que surgió y dio forma a los intereses de Estados Unidos para encontrar una solución. ${ }^{44}$ Finalmente, era menos probable que los golpes de Estado recibieran atención si los países involucrados eran económica y militarmente fuertes o si tenían poderosos aliados internacionales en el Consejo de Seguridad (por ejemplo, los casos de Egipto, Tailandia y Pakistán).

El Consejo de Seguridad es un órgano importante de la cara intergubernamental de la onu, y no sorprende que sus acciones reflejen los intereses de los Estados más poderosos que la integran. En contraste, los otros órganos fundamenta-

${ }^{43}$ Binder, "Paths to Intervention...", art. cit.

${ }^{44}$ D. M. Malone, Decision-making in the UN Security Council: The Case of Haiti, 1990-1997, Oxford, University Press, 1998. 
les de la ONU (especialmente la Secretaría y el Secretario General) operan de acuerdo con un conjunto diferente de imperativos políticos y algunas veces pueden desempeñar un papel independiente en la promoción de normas y de su institucionalización. ${ }^{45}$ El Secretario General tiene una función delicada en las Naciones Unidas, de alguna manera independiente de los Estados miembros, pero constreñido al mismo tiempo por los intereses y preferencias de éstos. Como han señalado varios estudiosos, mientras que los poderes formales de la posición son bastante limitados, la Carta establece más un papel político que uno puramente administrativo, y los secretarios generales individuales han utilizado con pericia, y de manera influyente, sus poderes formales e informales. ${ }^{46}$ Por lo tanto, el Secretario General tiene cierto margen de independencia y autonomía, pero no puede hacer caso omiso de los Estados miembros y, a menudo, entra en conflicto con ellos cuando divergen los intereses y las ideas.

Sin embargo, los secretarios generales individuales pueden desempeñar un papel esencial en la definición y promoción de los principios y objetivos de las Naciones Unidas, según diversos intereses, capacidades y compromisos personales. ${ }^{47}$ Por ejemplo, en la política y práctica de la onU sobre temas relativos a la democracia, el Secretario General Boutros Boutros-Ghali ha sido identificado como promotor de normas fundamentales en los años que ocupó el cargo, desde 1992 hasta 1996. Boutros-Ghali fue la fuerza impulsora detrás de la Agenda de Democratización ${ }^{48}$ de la onu y tuvo una

${ }^{45}$ Rushton, "The UN Secretary-General...", art. cit.; Krook y True, "Rethinking the Life Cycles", art. cit.

${ }^{46}$ S. Chesterman, ed., Secretary or General? The UN Secretary-General in World Politics, Cambridge, Cambridge University Press, 2007; K. J. Kille, From Manager to Visionary: The Secretary-General of the United Nations, Londres, Palgrave Macmillan, 2016.

${ }^{47}$ Edward Newman, "Secretary-General", in Sam Daws and Thomas G. Weiss, eds., The Oxford Handbook on the United Nations, Oxford: Oxford University Press, 2007.; Kille, From Manager to Visionary, op. cit.

${ }^{48}$ Rushton, "The UN Secretary-General and Norm Entrepreneurship", art. cit.; Lombardo, "Making of an Agenda for Democratization", art. cit. 
función importante en la configuración de la enérgica respuesta de la organización a las autoridades que sucedieron al golpe de Estado en Haití. ${ }^{49}$ Kofi Annan también buscó hacerse de un papel de liderazgo en temas de promoción de la democracia, en particular de la norma que se oponía al golpe de Estado. En la semana posterior al golpe en Sierra Leona, en 1997, que tuvo lugar durante su primer año en el cargo, Annan trató explícitamente de impulsar un nuevo principio antigolpista. Ante la Organización de la Unidad Africana (ouA), Annan declaró: "Dediquémonos a una nueva doctrina para la política africana: donde la democracia se ha usurpado, hagamos lo que esté a nuestro alcance para devolverla a sus legítimos dueños, el pueblo". ${ }^{50}$

En varias ocasiones, los secretarios generales subsiguientes se han manifestado con mayor firmeza que los organismos intergubernamentales de las Naciones Unidas, y con frecuencia han sido la única voz en la onU que reacciona ante golpes de Estado. Cuando el jefe de las fuerzas armadas de Pakistán tomó el poder en 1999, el Consejo de Seguridad permaneció en silencio, no sólo por el hecho de que Estados Unidos fuera antiguo aliado del país. Por el contrario, Annan emitió una declaración en la que deploraba el golpe e instaba a los militares a restablecer el gobierno civil. ${ }^{51}$ Otros casos en que el Secretario General se pronunció, pero ante los cuales el Consejo permaneció inmutable, incluyen varios golpes de Estado durante el mandato de Annan (Costa de Marfil en 1999, Fiji en 2000, República Centroafricana en 2003, Togo en 2005 y Mauritania en 2005), así como varios golpes de Estado que coincidieron con el mandato de Ban Ki-moon (Níger en 2010, Maldivas en 2012 y Egipto en 2013). Sin em-

${ }^{49}$ Malone, Decision-making in the UN Security Council.

${ }^{50}$ Discurso de Kofi Annan en la Asamblea Anual de Jefes de Estado y de Gobierno de la Organización de la Unidad Africana (OUA), 2 de junio de 1997.

51 Secretario General de la ONU, "Secretary-General Deplores Military Coup In Pakistan, Urges Early Restoration Of Civilian Rule”, comunicado de prensa, sG/sm/7174, 13 de octubre de 1999. 
bargo, al igual que con el Consejo de Seguridad, las declaraciones del Secretario General también pueden variar en lo que respecta a la severidad de su discurso, que va desde la condena absoluta de los golpes de Estado hasta las reprensiones más leves, como la respuesta al golpe de Estado de Egipto en 2013, ante el cual Ban sólo expresó "preocupación” acerca de la "injerencia militar". 52

Los registros sugieren que si bien los secretarios generales individuales suelen estar dispuestos a enunciar su opinión cuando el Consejo de Seguridad permanece en silencio, en la práctica a menudo se han visto limitados en casos esenciales en los que el golpe en cuestión tuvo lugar en un Estado regionalmente poderoso o en uno con poderosos aliados internacionales entre los miembros permanentes del Consejo de Seguridad. Aunque con mayor voz de mando que el Consejo de Seguridad, la oficina del Secretario General también muestra gran incongruencia en la aplicación de la norma antigolpista.

ESTRATEGIAS DE INSTITUCIONALIZACIÓN DE NORMAS eN la Secretaría de las Naciones Unidas

A principios de la década del 2000, varios funcionarios encumbrados de la Secretaría, en particular los que trabajaban en el Departamento de Asuntos Políticos (DPA, por sus siglas en inglés) de la onU, intentaron lograr un cambio de enfoque al institucionalizar aún más la norma antigolpista en el sistema de la ONU. Los esfuerzos culminaron en una reforma a las normas y procedimientos de la Secretaría en 2009, diseñada para generar una respuesta más consecuente y menos ad hoc a los golpes de Estado en todo el sistema de las Naciones Unidas.

52 Secretario General de la onu, "Secretary-General, Concerned About 'Military Interference' in Egypt, Stresses Need to Reinforce Civilian Rule, Peacefully Seek Common Ground Towards Transition”, comunicado de prensa, SG/sm/15151-AFr/2657, 3 de julio de 2013. 
Una serie de motivaciones dio impulso a tal empeño, pero en el centro estaba la insatisfacción con la incongruencia de los esfuerzos de la onU para hacer cumplir la norma. De acuerdo con varios funcionarios de la onU, había un sentimiento creciente en la Secretaría de que el enfoque de las Naciones Unidas para responder a los golpes de Estado se había caracterizado por políticas ad hoc y carentes de sistematización, ${ }^{53}$ lo cual se vio exacerbado por la percepción en 2008 y 2009 de que los golpes estaban, nuevamente, en aumento. Aunque el patrón general que siguió a la Guerra Fría ha implicado una pronunciada disminución en el número de golpes de Estado en comparación con décadas anteriores, hubo un aumento mínimo de golpes en África entre agosto de 2008 y marzo de 2009, que incluyeron a Guinea, Mauritania y Madagascar, seguidos rápidamente por el golpe en Honduras en junio de 2009. En abril de 2009, la Unión Africana anunció una decisión en que manifestaba preocupación por el resurgimiento de los golpes, y Uganda incorporó el tema a la agenda del Consejo de Seguridad de la onu, que de inmediato emitió una declaración presidencial en que expresaba una preocupación semejante y acogía con satisfacción las iniciativas de la UA en el área. ${ }^{54}$ El caso de Madagascar planteó problemas particulares en la onU, ya que las autoridades que sucedieron al golpe (encabezadas por Andry Rajoelina) fueron invitadas por la Secretaría de la onU a una conferencia de donantes en favor de Somalia en abril de 2009, lo que llevó a la uA a comunicar su descontento a la oficina del Secretario General de que la onU estaba minando los esfuerzos de la Unión Africana para aislar a las nuevas autoridades de facto. Este episodio aumentó la percepción en la Secretaría de la onu (en el Departamento de Asuntos Políticos y en la Oficina Ejecutiva del

53 Varios funcionarios de la Secretaría de la onu en el Departamento de Asuntos Políticos (DAP) y la Oficina Ejecutiva del Secretario General, entrevistados por el autor, sede de la onU, Nueva York, octubre de 2015.

54 Consejo de Seguridad de la onu, Declaración de la Presidencia S/PRST/2009/11; Informe del Consejo de Seguridad de la OnU, "The Resurgence of Coups d'état in Africa”, 15 de abril de 2009. 
Secretario General) de que era necesario tomar medidas para garantizar que las Naciones Unidas actuaran con mayor firmeza y coherencia en lo que atañe a los golpes de Estado y de que era problemático que se las viera relegadas, detrás de las organizaciones regionales. ${ }^{55}$

Además, había la sensación de que las diversas agencias internas de las Naciones Unidas también actuaban con propósitos contrarios en su respuesta a los golpes de Estado que se estaban suscitando. En particular, algunos funcionarios del DAP expresaron su frustración por el hecho de que otras ramas de la onU estaban siguiendo un enfoque tradicional y rutinario en los países que experimentaron golpes de Estado, en lugar de detenerse a reconsiderar las relaciones con los gobiernos que habían llegado al poder por medios inconstitucionales. ${ }^{56} \mathrm{Al}$ resumir el sentimiento general dentro de la onU en ese momento, uno de sus funcionarios eminentes mencionó que "había la sensación de que los golpes seguían ocurriendo con un ritmo constante y de que no éramos coherentes en nuestra respuesta; ciertamente, había que hacer conciencia, con la uA por delante de nosotros, de que debíamos hacer lo posible para tener más principios, más coherencia". 57

Sin embargo, también se reconoció en la Secretaría que el potencial de institucionalización de la norma estaba limitado por la posición de los Estados miembros. ${ }^{58}$ Las discusiones sobre la declaración presidencial en abril de 2009 confirmaron que los miembros permanentes clave del Consejo de Seguridad se resistían a cualquier movimiento que hiciera de la reprobación de los golpes de Estado una práctica automática

${ }^{55}$ Funcionario superior del DAP, comunicación personal con el autor, 17 de septiembre de 2015.

${ }^{56}$ Funcionarios del DAP, entrevistados por el autor, sede de la ONU, Nueva York, 14 de octubre de 2015.

${ }^{57}$ Funcionario superior de la Secretaría, entrevistado por el autor, sede de la onu, Nueva York, 6 de octubre de 2015.

${ }^{58}$ Funcionarios actuales, y antiguos, del DAP, entrevistados por el autor, sede de la onU, Nueva York, 5-15 de octubre de 2015. 
y que preferían, en cambio, la flexibilidad política para tratar caso por caso. ${ }^{59}$ No había "apetito" entre los miembros de la oNu por el tipo de instrumentos legales sólidos que se había adoptado en la OEA y en la UA y que especificaría causas inalterables para violaciones de normas claramente delineadas. ${ }^{60}$ En consecuencia, los artífices o promotores de estatutos en la Secretaría de la onU no intentaron impulsar la institucionalización de la norma antigolpista mediante la acción de los Estados miembros, sino que buscaron institucionalizarla aún más dentro de la misma Secretaría. La selección del lugar adquirió importancia, toda vez que tenía implicaciones significativas para la naturaleza y el grado de institucionalización de la norma a la que podría aspirarse de manera realista. ${ }^{61}$

El lugar decisivo para llevar adelante este empeño fue el Comité de Políticas (CP) de la Secretaría, que esencialmente funcionó como gabinete del Secretario General al reunir a funcionarios encumbrados de los organismos fundamentales de la Secretaría para organizar asambleas semanales sobre estrategia y política. La agenda del CP está determinada por la Secretaría, de modo que sus funcionarios de rango medio son quienes pueden presionar para incluir en la discusión temas específicos. Por lo tanto, el CP dio a quienes emprenden las normas en la ONU un lugar para promover sus disposiciones predilectas al más alto nivel, además de dar forma al tipo de institucionalización que podría buscarse. Si bien la Asamblea General y el Consejo de Seguridad pueden aprobar resoluciones que constriñen (y que a veces legalmente obligan) a los Estados miembros, el cP no está diseñado para dar forma a su política, sino a la política y estrategia de la propia Secretaría. El tipo de institucionalización disponible

${ }^{59}$ Informe del Consejo de Seguridad de la ONU, "The Resurgence of Coups D'état in Africa”, art. cit.

${ }^{60}$ Funcionario superior del DAP, entrevistado por el autor, sede de la ONU, Nueva York, 13 de octubre de 2015.

61 K. P. Coleman, "Locating Norm Diplomacy: Venue Change in International Norm Negotiations", European Journal of International Relations 19, núm. 1, 2013, pp. 163-186, doi: 10.1177/1354066111411209 
mediante el CP atañe a las reglas internas, procesos y procedimientos operativos comunes a que dan seguimiento los burócratas de las Naciones Unidas. Quienes emprenden las normas en la ONU aspiraron a una forma de institucionalización del mínimo común denominador; es decir, intentaron un cambio en el nivel más alto donde sospechaban que podían alcanzar logros tangibles, incluso si eso suponía llegar a un nivel de progreso más bajo de lo que idealmente se hubiera deseado.

En 2009, los funcionarios del Departamento de Asuntos Políticos (DAP) respondieron con extremada atención a las políticas de golpe de Estado de la ONU, al incluir el tema en la agenda del Comité de Políticas. En noviembre de 2009, el CP consideró una presentación de política, preparada por funcionarios de la DAP, sobre el tema "Respuesta de la ONU a cambios de gobierno inconstitucionales". ${ }^{62}$ La presentación sugirió que el patrón de los recientes golpes de Estado en África, así como los golpes en Honduras, Tailandia y Fiji, sirvieron para "plantear nuevas preguntas a la ONU sobre cómo responder a los cambios de gobierno inconstitucionales”. El documento observaba que los recientes golpes de Estado "ponen de relieve la creciente brecha normativa entre muchas organizaciones regionales (y subregionales) con estructuras de principios y la onU, que continúa respondiendo a los golpes de manera ad hoc". Este enfoque ad hoc, advertía, condujo por momentos a "mensajes contradictorios, oportunidades malogradas, aumento en los costos de transacción y dificultades que podían evitarse". Para encarar el problema, pidió al Secretario General "fomentar una postura que se basara en normas y principios sobre cambios de gobierno inconstitucionales", y recomendó que se iniciara un proceso mediante el cual la Secretaría (y el DAP en particular) comen-

${ }^{62}$ Naciones Unidas, "UN Response to Unconstitutional Change of Government: Submission to the Policy Committee", documento interno de la ONU, 2009. El autor principal de la presentación fue el entonces funcionario del DAP, Sebastian von Einsiedel. 
zaría un procedimiento común en respuesta a todos los golpes de Estado. El documento reconoció que en los órganos intergubernamentales de las Naciones Unidas había un limitado margen para avanzar en el tema y, en cambio, destacó la capacidad de la Secretaría para tomar iniciativas en tales dominios.

La recomendación fundamental de la presentación de la política sugería que, a raíz de cualquier golpe, se convocaría un grupo de trabajo interdepartamental para coordinar las respuestas públicas de las Naciones Unidas, proporcionar orientación sobre políticas en todo el sistema de la onU y, lo que es más importante, supervisar la coordinación con socios internacionales que no pertenecieran a las Naciones Unidas. También se abogó por que el Secretario General tomara una vía más sistemática en las respuestas públicas y se recomendó dar un "mensaje claro de desaprobación" para presionar a las autoridades de facto en el país en cuestión y enviar una señal a toda la onU de que las cosas no podían transcurrir como si todo rampara en la normalidad. Es importante destacar que la presentación también planteó la posibilidad de emitir más sanciones contra los líderes golpistas, incluido el hecho de que no se les invite a conferencias fuera de los organismos intergubernamentales, ${ }^{63}$ que se suspenda la asistencia electoral y para el desarrollo, y que la onU revise cualquier contribución al mantenimiento de la paz de los países liderados por golpistas. Debido a que los Estados miembros reciben ciertas retribuciones de la onU por cooperar en las maniobras de mantenimiento de la paz, la suspensión de tales contribuciones podría servir como una poderosa herramienta de influencia política.

Después de considerar la presentación en noviembre de 2009, el cP adoptó la Decisión PC/2009/24, que comprometió al Secretario General a promover, según cité más arriba,

63 Los Estados miembros manejan su propia acreditación formal en el Comité de Credenciales de la Asamblea General, por lo que la Secretaría no está en condiciones de suspender a un país de la onU. 
"una postura que se basara en normas y principios sobre cambios de gobierno inconstitucionales". La decisión del CP también adoptó algunas de las recomendaciones de fondo que contenía la presentación de la DAP. El aspecto más concreto de la Decisión PC/2009/24 trajo consigo la constitución de un grupo de trabajo interdepartamental que pudiera convocarse dentro de las 24 horas posteriores a cualquier cambio de gobierno inconstitucional para coordinar la respuesta inicial de la onU y brindar consejo y orientación diaria sobre políticas. Este grupo también ofrecería instrucción acerca de las declaraciones del Secretario General, la coordinación entre los organismos de las Naciones Unidas y la presencia de la organización en el terreno y la coordinación con sus socios internacionales. Además, daría asesoramiento acerca de las situaciones en que la Secretaría debe coincidir con las autoridades de facto. Respecto de las opciones políticas más punitivas, planteadas por la presentación de políticas del DAP, incluidas las posibles modificaciones en las políticas de apoyo electoral, la asistencia para el desarrollo y las contribuciones para el mantenimiento de la paz, la Decisión PC/2009/24 simplemente propuso que el Secretario General convocara al CP para determinar una respuesta estratégica. Además de requerir la creación de un grupo de trabajo después de cada golpe, la Decisión no especificó ninguna respuesta política en particular ni institucionalizó sanción alguna contra los líderes golpistas. Esta relativa debilidad de la Decisión reflejó los desacuerdos entre las agencias de la onU sobre el alcance de la acción punitiva que el Secretario General podía, y debía, imponer a los países que pasaban por una experiencia golpista. La Oficina de Asuntos Jurídicos de la onU tiene una visión minimalista de la autoridad del Secretario General y, ante la ausencia de un consenso de los Estados miembros sobre el tema, se resistió a los esfuerzos por establecer un papel más intervencionista que incluiría la imposición de sanciones costosas por el Secretario General a Estados miembros 
individuales. ${ }^{64}$ El Departamento de Operaciones de Mantenimiento de la Paz (DPKO, por sus siglas en inglés) también se resistió a cualquier sugerencia que impidiera automáticamente a los países golpistas contribuir con tropas a las operaciones destinadas a mantener la paz. En un momento en que los compromisos de preservación de la paz de la onu se encontraban en niveles sin precedentes, el DPKo intentó evitar cualquier política que redujera de manera automática el número de tropas que tenía a su disposición para la tarea de custodiar la paz. ${ }^{65}$

La Decisión PC/2009/24 creó así nuevas reglas y procedimientos que el personal de la onU debía seguir después de un golpe de Estado, sin idear instrumentos punitivos específicos ni automáticos. El resultado del proceso reflejó las opciones limitadas y disponibles para los funcionarios de la Secretaría. Ante la falta de apoyo de los Estados miembros para una mayor institucionalización de las normas, los artífices de éstas en las Naciones Unidas procuraron lograr lo que podían y donde podían. A diferencia del Consejo de Seguridad, el lugar que eligieron no tenía la autoridad para legalizar las normas antigolpistas con instrumentos vinculantes que exigirían de los Estados miembros responder con firmeza a todo episodio que tuviera por protagonista algún golpe de Estado. Sin embargo, el Comité de Políticas dio la oportunidad de institucionalizar reglas y procedimientos dentro de la Secretaría que aumentaron las perspectivas de otras formas de coherencia, particularmente haciendo que el sistema de las Naciones Unidas se expresara con unanimidad y asegurando que éstas aportaran su visión de los golpes de Estado individuales de manera más coincidente con otros actores internacionales. Tal como lo comentó un funcionario de alto rango del DAP sobre la decisión del CP, el "contenido era

${ }^{64}$ Funcionarios de la Oficina de Asuntos Jurídicos (ola, según sus siglas en inglés) y del DAP, entrevistados por el autor, sede de la ONU, Nueva York, 12 de octubre de 2015.

${ }^{65}$ Funcionario del DAP, entrevistado por el autor, sede de la ONU, Nueva York, octubre de 2015. 
procesal". ${ }^{66}$ Los nuevos procedimientos se diseñaron para fomentar una mayor coordinación dentro del sistema de la ONU, de modo que los departamentos individuales dentro de la Secretaría aclararan su propia postura y la compartieran con otros departamentos y entidades del sistema, con el propósito de afirmarse en una postura coherente que después pudieran compartir, además, con los socios internacionales de relevancia. ${ }^{67}$

Para aquellos que introdujeron la norma antigolpista en la agenda de la ONU, el resultado fue el mejor que podía lograrse. En un comentario que secundaron otros participantes en el proceso, uno de los artífices esenciales del esfuerzo por institucionalizar aún más la norma declaró: "obtuvimos lo que buscábamos" ${ }^{68}$ La Decisión PC/2009/24 ayudó a reducir, si no a eliminar, algunas de las incongruencias que habían impulsado el esfuerzo de reforma, para empezar. Las respuestas a los golpes de Estado se volvieron "menos ad hoc, con procedimientos más claros, más coherentes". ${ }^{69}$ Los funcionarios del DAP también informaron de un distanciamiento de la visión indiferente de antaño entre algunas agencias de la onu que habían contribuido a la incongruencia entre actores diversos: "La política cambió lo único que se suponía que debía cambiar, esto es forzar una reflexión sistémica sobre "¿qué hacemos ahora que está sucediendo algo diferente?»".70 La incongruencia no se eliminó, pero el es-

${ }^{66}$ Funcionario superior del DAP, entrevistado por el autor, sede de la ONU, Nueva York, octubre de 2015.

${ }^{67}$ Funcionario del DAP, entrevistado por el autor, sede de la ONU, Nueva York, octubre de 2015.

${ }^{68}$ Funcionario superior del DAP, entrevistado por el autor, sede de la ONU, Nueva York, 13 de octubre de 2015. Otro funcionario del DAP, que contribuyó a impulsar la reforma, dijo: "Finalmente, obtuvimos lo que queríamos". Este mismo funcionario fue entrevistado por el autor, sede de la onu, Nueva York, 13 de octubre de 2015.

${ }^{69}$ Funcionario superior del DAP, entrevistado por el autor, sede de la ONU, Nueva York, 13 de octubre de 2015.

${ }^{70}$ Funcionario del DAP, entrevistado por el autor, sede de la ONU, Nueva York, 14 de octubre de 2015. 
fuerzo por institucionalizar la norma antigolpista creó nuevas reglas y procedimientos que redujeron el alcance de los mensajes contradictorios y de los problemas evitables que habían caracterizado los enfoques anteriores.

\section{Conclusión}

Cuando los actores internacionales hacen cumplir las normas de manera selectiva, los artífices o emprendedores de normas internacionales a menudo intentan lograr una estrategia de institucionalización que aumente la aplicación coherente de éstas. Sin embargo, no todas las estrategias de institucionalización de normas son iguales, y los promotores deben elegir aquellas que tengan mayor posibilidad de éxito. Cuando los Estados no están de acuerdo con el valor de las normas individuales, las probabilidades de su institucionalización, sólida y profunda (por ejemplo, la introducción de instrumentos legalmente obligatorios), son inciertas, y es factible que a los casos de violación de la norma se den respuestas selectivas. En tales situaciones, quienes emprenden las normas pueden buscar la institucionalización en un nivel inferior, para rehuir los esfuerzos que implica la legalización de la norma y, en cambio, centrarse en normas y procedimientos menos vinculantes, pero con frecuencia muy influyentes. Estas estrategias pueden equivaler a la institucionalización de normas con el denominador común más bajo, en que los que promueven las normas aspiran a alcanzar el nivel más alto posible de institucionalización, incluso si tal logro significa que sus objetivos estratégicos se han desencaminado de lo que en principio constituía la meta. Cuando se persiguen estas formas de institucionalización en un nivel inferior, es menos probable que alcancen plena coherencia, ya que, ante la ausencia de normas legales obligatorias, los Estados continuarán siendo libres de responder según les dicte su voluntad. Sin embargo, estos esfuerzos pueden reducir la falta de sistematicidad de otras formas de aplicación, ya que las 
reglas reformadas y de nuevo cuño, además de los procedimientos operativos comunes, pueden asegurar que las agencias y el personal de una organización internacional se expresen al unísono y se coordinen entre sí, lo que disminuye el riesgo de que haya mensajes contradictorios dentro y entre organizaciones internacionales.

La fortuna de la norma antigolpista en las Naciones Unidas ilustra claramente estas dinámicas imperantes. Después de años de una aplicación de la norma antigolpe extremadamente incongruente, sus artífices trataron de que lograra una mayor institucionalización. Sin embargo, ante la falta de consentimiento entre los Estados miembros, evitaron cualquier esfuerzo por impulsar la introducción de normas legalmente obligatorias por medio del Consejo de Seguridad (un resultado que sería improbable) y, en su lugar, se concentraron en desarrollar normas y procedimientos de orden inferior para mejorar la coordinación dentro de la burocracia de la ONU, así como entre ésta y otras organizaciones internacionales. La estrategia elegida no se diseñó para garantizar que los principales órganos intergubernamentales de la onU respondieran por igual a todos los golpes de Estado, sino para reducir el alcance de los indicios de enfrentamiento desde dentro del conjunto del sistema de la onU. La evolución de la norma antigolpista en la organización pone en evidencia los diversos tipos de aplicación de normas incongruentes que puede haber y el potencial (y los límites) de las diferentes estrategias de institucionalización a que pueden aspirar los promotores para lograr un enfoque más sistemático ante las violaciones a la norma en cuestión.

Traducción de JoRge VAlenzuela

BiBLIOGRAFÍA

Аввотт, Kenneth W., Robert O. Keohane, Andrew Moravcsik, AnneMarie Slaughter y Duncan Snidal, "The Concept of Legaliza- 
tion”, International Organization 54, núm. 3, 2000, pp. 401-419, doi: $10.1162 / 002081800551271$

Аввотт, Kenneth W. y Duncan Snidal, "Why States Act Through Formal International Organizations", Journal of Conflict Resolution 42, núm. 1, 1998, pp. 3-32, doi: 10.1177/00220027980420 01001

Annan, Kofi, Discurso en la Asamblea Anual de Jefes de Estado y de Gobierno de la Organización de la Unidad Africana, ouA, 2 de junio de 1997.

BARNETT, Michael y Martha Finnemore, Rules for the World: International Organizations in Global Politics, Ithaca, Cornell University Press, 2004.

Beardsley, Kyle y Holger Schmidt, "Following the Flag or Following the Charter? Examining the Determinants of un Involvement in International Crises, 1945-20021", International Studies Quarterly 56, núm. 1, 2012, pp. 33-49.

Billerbeck, Sarah von, "Local Ownership and UN Peacebuilding: Discourse Versus Operationalization”, Global Governance: A Review of Multilateralism and International Organizations 21, núm. 2, 2015, pp. 299-315.

Binder, Martin, "Humanitarian Crises and the International Politics of Selectivity", Human Rights Review 10, núm. 3, 2009, pp. 327-148, doi: 10.1007/s12142-009-0121-7

Binder, Martin, "Paths to Intervention: What Explains the Un's Selective Response to Humanitarian Crises?” Journal of Peace Research 52, núm. 6, 2015, pp. 712-726, doi: 10.1177/00 223433 15585847

Björkdahl, Annika, "Promoting Norms Through Peacekeeping: UNPREDEP and Conflict Prevention", International Peacekeeping 13,núm. 2,2006,pp.214-228, doi:10.1080/13533310500437613

Carothers, Thomas, "The Backlash Against Democracy Promotion”, Foreign Affairs 85, núm. 2, 2006, pp. 55-68

Chesterman, Simon, Just War or Just Peace? Humanitarian Intervention and International Law, Oxford, University Press, 2003.

Chesterman, Simon (ed.), Secretary or General? The un Secretary-General in World Politics, Cambridge, University Press, 2007. 
Coleman, Katharina P., "Locating Norm Diplomacy: Venue Change in International Norm Negotiations", European Journal of International Relations 19, núm. 1, 2013, pp. 163-186, doi: 10.11 $77 / 1354066111411209$

Cooley, Alexander, "Countering Democratic Norms", Journal of Democracy 26, núm. 3, 2015, pp. 49-63.

Donno, Daniela, Defending Democratic Norms: International Actors and the Politics of Electoral Misconduct, Oxford, University Press, 2013.

Einsiedel, Sebastian von, David M. Malone y Bruno Stagno Ugarte, The Un Security Council in the 21st Century, Boulder, Lynne Rienner, 2015.

Finnemore, Martha, "International Organizations as Teachers of Norms: The United Nations Educational, Scientific, and Cultural Organization and Science Policy", International Organization 47, núm. 4, 1993, pp. 565-597, doi: 10.1017/S0020 8183000 28101

Finnemore, Martha y Kathryn Sikkink, "International Norm Dynamics and Political Change", International Organization 52, núm. 4, 1998, pp. 887-917.

Goldstein, Judith, Miles Kahler, Robert O. Keohane y Anne-Marie Slaughter, "Introduction: Legalization and World Politics", International Organization 54, núm. 3, 2000, pp. 387, doi: 10.11 $62 / 002081800551262$

HaAck, Kirsten, The United Nations Democracy Agenda: A Conceptual History, Oxford, University Press, 2011.

Hafner-Burton, Emilie M., Susan D. Hyde y Ryan S. Jablonski, "When Do Governments Resort to Election Violence?", British Journal of Political Science 44, núm. 1, 2013, pp. 1-31.

Haftel, Yoram Z. y Alexander Thompson, "The Independence of International Organizations: Concept and Applications", Journal of Conflict Resolution 50, núm. 2, 2006, pp. 253-275, doi: $10.1177 / 0022002705285288$

Hawkins, Darren, "Protecting Democracy in Europe and the Americas", International Organization 62, núm. 3, 2008, pp. 373-403. 
Heine, Jorge y Brigitte Weiffen, Twenty-first Century Democracy Promotion in the Americas: Standing Up for the Polity, Londres, Routledge, 2014.

Joyner, Christopher C., "The United Nations and Democracy", Global Governance 5, núm. 3, 1999, pp. 333-357

Kelley, Judith, "D-minus Elections: The Politics and Norms of International Election Observation", International Organization 63, núm. 4, 2009, pp. 765-787, doi: 10.1017/S002081830999 0117

Kille, Kent J., From Manager to Visionary: The Secretary-General of the United Nations, Londres, Palgrave Macmillan, 2016.

Kratochwil, Friedrich V., Rules, Norms, and Decisions: On the Conditions of Practical and Legal Reasoning in International Relations and Domestic Affairs, vol. 2, Cambridge, University Press, 1991.

Квоок, Mona Lena y Jacqui True, "Rethinking the Life Cycles of International Norms: The United Nations and the Global Promotion of Gender Equality", European Journal of International Relations 18, núm. 1, 2012, pp. 103-127.

Legler, Thomas y Thomas Kwasi Tieku, "What Difference Can a Path Make? Regional Democracy Promotion Regimes in the Americas and Africa", Democratization 17, núm. 3, 2010, pp. 465-491.

Leininger, Julia, "Against All Odds: Strong Democratic Norms in the African Union", en Tanja A. Börzel y Vera van Hüllen (eds.), Governance Transfer by Regional Organizations, Nueva York, Springer, 2015, pp. 51-67, en http://link.springer.com/ chapter/10.1057/9781137385642_3

Lombardo, Caroline E., "The Making of an Agenda for Democratization: A Speechwriter's View", Chicago Journal of International Law 2, núm. 1, 2001, pp. 253-266.

Malone, David M. Decision-making in the UN Security Council: The Case of Haiti, 1990-1997, Oxford, University Press, 1998.

Nathan, Laurie, "Trends in Mediating in Africa Coups, 20002015”, investigación presentada en la International Studies Association Annual Convention, 2016.

Percy, Sarah, Mercenaries: The History of a Norm in International Relations, Oxford, University Press, 2007. 
Pevehouse, Jon C., Democracy from Above: Regional Organizations and Democratization, Cambridge, University Press, 2005.

Powell, Jonathan y Clayton Thyne, "Global Instances of Coups from 1950 to 2010: A New Dataset”, Journal of Peace Research 48, núm. 2, 2011, pp. 249-259, doi: 10.1177/0022343310397436

Powell, Jonathan, Trace Lasley y Rebecca Schiel, "Combating Coups d'État in Africa, 1950-2014”, Studies in Comparative International Development 56, núm. 6, 2016, pp. 1-21, doi: 10. 1007/ s12116-015-9210-6

Rushton, Simon, "The UN Secretary-General and Norm Entrepreneurship: Boutros Boutros-Ghali and Democracy Promotion”, Global Governance: A Review of Multilateralism and International Organizations 14, núm. 1, 2008, pp. 95-110

Shraga, Daphna, "The Security Council and Human Rights From Discretion to Promote to Obligation to Protect", en Bardo Fassbender (ed.), Securing Human Rights? Achievements and Challenges of the UN Security Council, Oxford, University Press, n.d., pp. 16-17.

SOUARÉ, Issaka K., "The AU and the Challenge of Unconstitutional Changes of Government in Africa”, Institute for Security Studies Papers, núm. 197, 2009, pp. 1-13.

Tansey, Oisín, "The Fading of the Anti-coup Norm", Journal of Democracy 28, núm. 1, 2017, pp. 144-156, doi: 10.1353/jod.2017. 0012

Tansey, Oisín , "The Limits of the 'Democratic Coup' Thesis: International Politics and Post-coup Authoritarianism", Journal of Global Security Studies 1, núm. 3, 2016, pp. 220-234.

White, Nigel D., "The United Nations and Democracy Assistance: Developing Practice Within a Constitutional Framework", en Peter Burnell, (ed.), Democracy Assistance: International Co-operation for Democratization, Londres, Frank Cass, 2000, pp. 67-89

Instrumentos jurídicos, informes y reportes

Asamblea General de las Naciones Unidas, Res. 46/7, 11 de octubre de 1991. 
Asamblea General de las Naciones Unidas, Res. 63/301, 1 de julio de 1999.

Comunicado de prensa del Consejo de Seguridad de la onu sobre Guinea-Bisáu sc/7873, 15 de septiembre de 2003

Comunicado de prensa del Consejo de Seguridad de la onu, sc/8 894, 7 de diciembre de 2006.

Consejo de Seguridad de la onu, Res. s/Res/940, 31 de julio de 1994.

Consejo de Seguridad de la onu, Res. s/REs/1072, 30 de agosto de 1996.

Consejo de Seguridad de la onu, Declaración de la Presidencia s/prst/1997/37, 11 de julio de 1997.

Consejo de Seguridad de la onu, Res. s/REs/1132, 8 de octubre de 1997.

Consejo de Seguridad de la onu, Declaración de la Presidencia S/PRST/2008/30, 19 de agosto de 2008.

Consejo de Seguridad de la onu, Declaración de la Presidencia S/PRST/2009/11.

Consejo de Seguridad de la onu, Declaración de la Presidencia S/PRST/2012/15, 21 de abril de 2012.

Consejo de Seguridad de la onU, Res. S/REs/2048, 18 de mayo de 2012.

Informe del Consejo de Seguridad de la onu, "The Resurgence of Coups D'état in Africa”, 15 de abril de 2009, en www.securi tycouncilreport.org

Informe del Consejo de Seguridad de la ONU, "The Resurgence of Coups D'état in Africa".

Naciones Unidas (ONU), "UN Response to Unconstitutional Change of Government: Submission to the Policy Committee", documento interno de la onU, 2009. El autor principal de la presentación fue el entonces funcionario del DAP, Sebastian von Einsiedel.

Secretario General de la ond, "Secretary-General Deplores Military Coup In Pakistan, Urges Early Restoration Of Civilian Rule”, comunicado de prensa, SG/sm/7174, 13 de octubre de 1999.

Secretario General de la onu, "Secretary-General, Concerned About 'Military Interference' en Egypt, Stresses Need to Rein- 
force Civilian Rule, Peacefully Seek Common Ground Towards Transition", comunicado de prensa, sG/sm/15151-AFR/2657, 3 de julio de 2013.

Entrevistas y comunicaciones personales

Varios funcionarios actuales y antiguos en septiembre y octubre de 2015 en la sede de la Organización de las Naciones Unidas en Nueva York:

Secretaría de la onu en el Departamento de Asuntos Políticos (DAP) Oficina de Asuntos Jurídicos (olA, por sus siglas en inglés)

Oficina Ejecutiva del Secretario General, sede de la onu, Nueva York, octubre de 2015. 